\title{
Wormholes supported by Scalar Fields with Negative Kinetic Energy
}

\author{
F.Rahaman*, M.Kalam ${ }^{\dagger}$ and K. A. Rahman*
}

\section{Abstract}

We provide a new model of higher dimensional wormholes supported by phantom energy derived from a scalar field with negative kinetic term. We have shown that the Averaged Null Energy Condition ( ANEC) violating phantom energy can be reduced as desired. We have also noticed that dimension of the spacetime plays a crucial role for measuring the ANEC violating matter needed.

\section{Introduction:}

During last five or six years, scientists around the world follow more or less the same path to explain the accelerating phase of the Universe and to describe the wormhole physics. The first one is an experimental evidence observed first by two group of scientists, Perlmutter et al [1] and Riess et

${ }^{0}$ Key words: Scalar field; Wormhole; Higher dimension.

*Dept.of Mathematics, Jadavpur University, Kolkata-700 032, India

E-Mail:farook_rahaman@yahoo.com

†Dept. of Phys. , Netaji Nagar College for Women, Regent Estate, Kolkata-700092, India.

E-Mail:mehedikalam@yahoo.co.in al [2] about 10 years ago. Whereas the second phenomena is a purely theoretical prediction, predicted by Morris and Thorne [3] about 20 years back. Almost all the authors believe that this acceleration is due to the cause of unknown dark energy. The exciting and unusual geometry ( hypothetical short cut in spacetime ) that aries as the solutions of Einstein's equations is the wormhole. The most disappointing fact that it needs unusual matter or energy ( termed as exotic matter ). But fortunately, the spheres of action of these heavenly sources ( dark energy and exotic matter ) are same i.e. to produce negative pressure. So wormhole physicists and cosmologists exchange their ideas to achieve their goals. Cosmologists have no headache, what amount of dark energy to be needed to fulfill their target. But wormhole physicists should have to keep in view the minimizing the total amount of exotic matter. Most of the works done considering the ideas of phantom energy characterized by the equation of state ( EOS ) $p=w \rho$, with $w<-1$, where, $p$ and $\rho$ are respectively, the pressure and energy density of the source and Chaplygin gas EOS characterized by $p=a \rho-\frac{b}{\rho^{\alpha}},(a, b$ and $\alpha$ are positive constants ) in their respective fields of interest (i.e. explaining the accelerating state of the Universe and discussing wormhole physics ) [4-20]. 
Recently, some cosmologists have considered phantom dark energy models with negative kinetic term to explain the accelerating phase of the universe[21-23].

In this work, we have borrowed from cosmologists the idea of phantom energy that is generated by a scalar field Lagrangian with a negative kinetic term. We have developed the higher dimensional wormhole geometry using the above phantom source. Due to the string theory the gravity is a truly higher dimensional interaction which becomes effectively four dimension at low energies. Newtonian theory can not be obtained as a limit of Einstein's theory. Wormhole structure can not be obtained from Newtonian theory. But it is argued that wormhole like geometry could be found in Einstein's theory. Moreover, to unify gravity with other fundamental forces in nature, many theories such as Super string theories, $\mathrm{M}$ theories etc demand extra spatial dimension to be consistent. For this reason, why we consider higher dimension to discuss wormhole structure. The plan of the article is as follows: In the second section, we have presented the basic equations for modelling the wormhole spacetime. In section three, we have obtained the solutions of the field equations. The properties of the wormholes are discussed in section four. Last section is devoted to a brief summary and discussions including the calculations of the total amount of average null energy condition violating exotic matter needed.

\section{Construction of wormholes:}

For the present study the metric for static spherically symmetric spacetime in higher dimension is taken as

$$
d s^{2}=-e^{2 \alpha(r)} d t^{2}+e^{2 \beta(r)} d r^{2}+r^{2} d \Omega_{d}^{2}
$$

where, The line element $d \Omega_{d}{ }^{2}$ on the unit $d$-sphere is given by

$$
\begin{array}{r}
d \Omega_{d}^{2}=d \theta_{1}^{2}+\sin ^{2} \theta_{1} d \theta_{2}{ }^{2}+\sin ^{2} \theta_{1} \sin ^{2} \theta_{2} d \theta_{3}^{2} \\
+\ldots \ldots \ldots \ldots . .+\prod_{n=1}^{d-1} \sin ^{2} \theta_{n} d \theta_{d}^{2}
\end{array}
$$

Now, we consider the model which contains a minimally coupled massless negative kinetic scalar field. Here the action is taken as

$$
S=\int d^{d+2} x \sqrt{-g}\left[\frac{R}{2 \kappa^{2}}-\frac{1}{2} g^{\mu \nu} \partial_{\mu} \phi \partial_{\nu} \phi\right]
$$

where, $\kappa^{2}=8 \pi G$. Here the scalar field configuration is static and spherically symmetric, $\phi_{\mu}$ can only have one non-zero component,$\phi_{\mu}=\phi^{\prime}$. Now, the energy momentum tensor components in the static wormhole have the form

$$
e^{2 \beta-2 \alpha} T_{t t}=T_{r r}=-\frac{1}{2}\left(\phi^{\prime}\right)^{2}
$$

The equation of motion for $\phi, \square^{2} \phi=0$ is

$$
\phi^{\prime \prime}+\left(\alpha^{\prime}-\beta^{\prime}+\frac{d}{r}\right) \phi^{\prime}=0
$$

For the metric (1) and using the above energy momentum tensor, the independent Einstein's equations are

$$
\begin{aligned}
& \frac{d}{r} \beta^{\prime}+\frac{d(d-1)}{2 r^{2}}\left(e^{2 \beta}-1\right)=-4 \pi G\left(\phi^{\prime}\right)^{2} \\
& \frac{d}{r} \alpha^{\prime}-\frac{d(d-1)}{2 r^{2}}\left(e^{2 \beta}-1\right)=-4 \pi G\left(\phi^{\prime}\right)^{2}
\end{aligned}
$$




\section{Solutions:}

The field equation (5) for $\phi$ yields

$$
\phi^{\prime}=\frac{\phi_{0} e^{\beta-\alpha}}{r^{d}}
$$

where, $\phi_{0}$ is an integration constant.

To control the solutions, we assume the following assumption:

$$
\alpha(r)=0
$$

Argument: One of the traversability properties is that the tidal gravitational forces experienced by a traveller must be reasonably small. So, we assume a zero tidal force as seen by the stationary observer. Thus one of the traversability conditions is automatically satisfied.

Using equations (8), (9), one gets the solutions from the field equations (6) and (7) as

$$
e^{-2 \beta}=1-\frac{b(r)}{r}
$$

where, the shape function $\mathrm{b}(\mathrm{r})$ is given by

$$
b(r)=\frac{8 \pi G \phi_{0}^{2}}{d(d-1) r^{2 d-3}}
$$

The corresponding scalar field solution is

$$
\phi=\frac{1}{(d-1) \sqrt{\frac{8 \pi G}{d(d-1)}}} \cos ^{-1} \sqrt{\frac{8 \pi G \phi_{0}^{2}}{d(d-1) r^{2 d-2}}}
$$

One can note that $\phi \rightarrow 0$ as $r \rightarrow r_{0}$ whereas $\phi$ tends to a constant value when $r>>r_{0}$.

\section{Wormhole structure:}

For the assumption $\alpha(r)=0$ implies no horizon exists in the spacetime. Also one can note that $\frac{b(r)}{r} \rightarrow 0$ as $|r| \rightarrow \infty$ i.e. the spacetime is asymptotically flat. Here the throat occurs at $r=r_{0}$ for which $b\left(r_{0}\right)=r_{0}$.

We notice that $b^{\prime}\left(r_{0}\right)<1$ implies $d>$ 1. Thus flare-out condition has been satisfied since our spacetime is greater than or equal to four dimension. Thus our solution describing a static spherically symmetric higher dimensional wormhole supported by scalar Fields with Negative Kinetic Energy.

The axially symmetric embedded surface $z=z(r)$ shaping the Wormhole's spatial geometry is a solution of

$$
\frac{d z}{d r}= \pm \frac{1}{\sqrt{\frac{r}{b(r)}-1}}= \pm \frac{1}{\sqrt{\frac{d(d-1) r^{2 d-2}}{8 \pi G \phi_{0}^{2}}-1}}
$$

One can note from the definition of Wormhole that at $r=r_{0}$ (the wormhole throat) Eq.(14) is divergent i.e. embedded surface is vertical there. 
According to Morris and Thorne [3] , the ' $r$ ' co-ordinate is ill-behaved near the throat, but proper radial distance

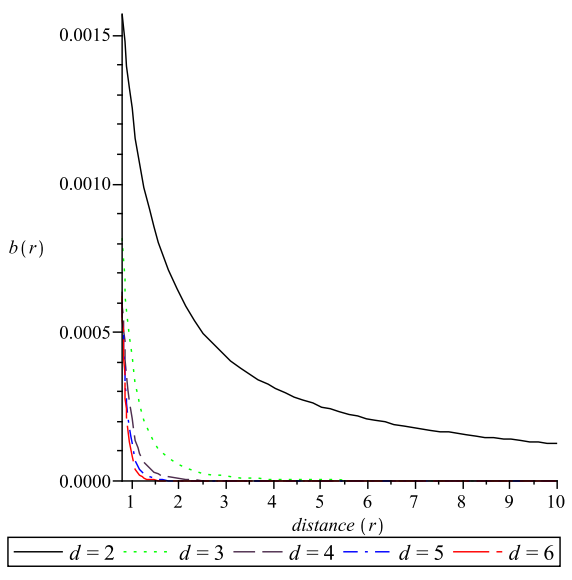

Figure 1: The diagram of the shape function of the wormhole for different dimensions $\left(\phi_{0}=.01, G=1\right)$.



Figure 2: The ratio $\frac{b(r)}{r}$ is plotted for different dimensions $\left(\phi_{0}=.01, G=1\right)$.

$$
l(r)= \pm \int_{r_{0}^{+}}^{r} \frac{d r}{\sqrt{1-\frac{b(r)}{r}}}
$$

must be well behaved everywhere i.e. we must require that $l(r)$ is finite throughout the space-time .

For our model, one can determine the proper distance through the wormhole as ( expanding binomially in powers of $\mathrm{r}$ and assuming $\phi_{0}^{4}$ and higher powers are zero )

$$
l(r)=\left[r-\frac{8 \pi G \phi_{0}^{2} r^{2 d-3}}{d(d-1)(2 d-3)}\right]
$$



Figure 3: The diagram of the radial proper distance of the wormhole for different dimensions $\left(\phi_{0}=.01, G=1\right)$. 


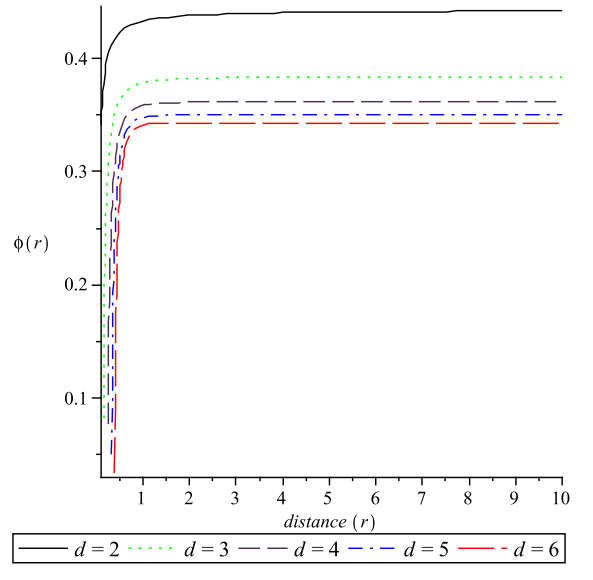

Figure 4: We plot the scalar field $\phi$ for different dimensions $\left(\phi_{0}=.01, G=1\right)$.

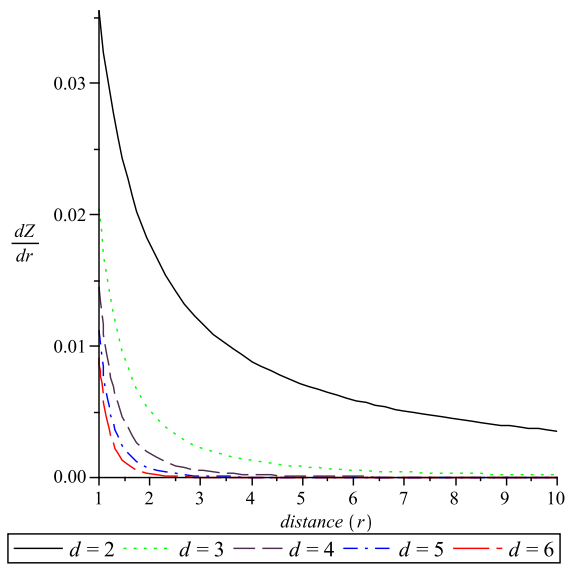

Figure 5: The nature of $\frac{d Z}{d r}$ for different dimensions $\left(\phi_{0}=.01, G=1\right)$.

\section{Summary and Discussions:}

In this article, we have presented a higher dimensional wormhole supported by phantom energy derived from a scalar field with negative kinetic term. In this model the exotic matter is described by a scalar field with negative kinetic Energy. The total amount of average null energy condition (ANEC) violating exotic matter for this wormhole can be quantified by the integral [24-25]

$$
\begin{aligned}
& I=\oint\left(T_{t}^{t}+T_{r}^{r}\right) d V \\
& \left.=\int_{r_{0}}^{\infty} 2\left[-\left(\phi^{\prime}\right)^{2}\right) e^{-2 \beta} \frac{2 \pi^{\frac{d+1}{2}}}{\Gamma\left(\frac{d+1}{2}\right)}\right] r^{d} d r \\
& =-\frac{4 \phi_{0}^{2} \pi^{\frac{d+1}{2}}}{\Gamma\left(\frac{d+1}{2}\right)(d-1) \sqrt{\frac{8 \pi G \phi_{0}^{2}}{d(d-1)}}} .
\end{aligned}
$$

Now, we are interested to the fact that under what conditions the total amount of ANEC violating matter could be reduced. One could see that $\phi_{0}$ and dimension of the spacetime affect the total amount of ANEC violating matter needed. The variation of the total amount of ANEC violating matter with respect to $\phi_{0}$ and dimension are shown in the figures 6-8. If the dimension of the spacetime is kept fixed, then the total amount of ANEC violating matter is reduced by decreasing $\phi_{0}$ as desired. It is surprising to note that the total amount of ANEC violating matter needed is decreasing with respect to dimension greater than eight but up to dimension eight it is increasing. The figures 6-8 support this astonishing phenomena. The assumption that the redshift function to be constant function implies the tidal gravitational force experienced by a traveller is zero. Thus one of the traversibility condition is satisfied, in other words, our wormholes containing small amount of exotic matter, in spite of, they are traversable for human beings. We have presented an example ( for four dimen- 
sional case ) of the possible structure of the wormhole generated by the rotation of the embedded curve about the vertical $\mathrm{z}$ axis (see appendix for details).

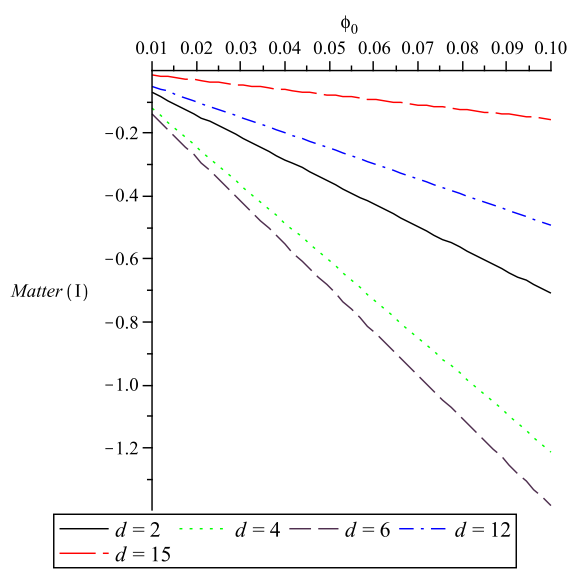

Figure 6: We show the variation of total amount of ANEC violating matter with respect to $\phi_{0}$ for different dimensions $(G=1$ ) .

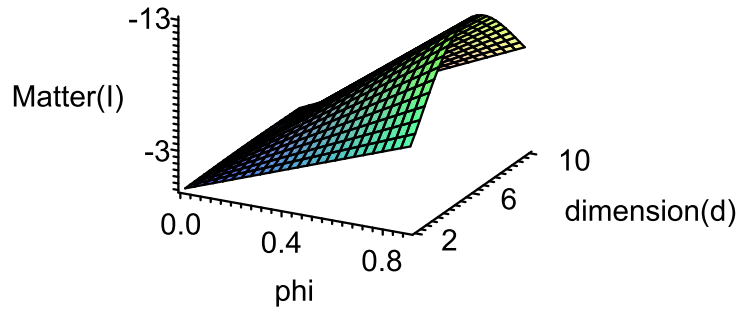

Figure 8: We show the variation of total amount of ANEC violating matter with respect to dimension and $\phi_{0}(G=1)$.

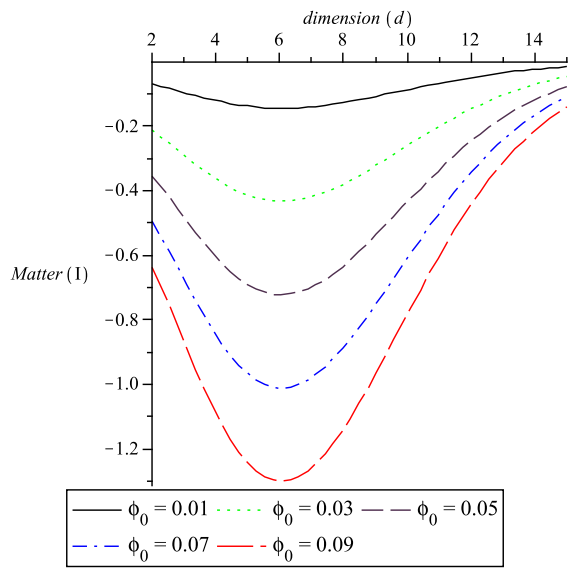

Figure 7: We show the variation of total amount of ANEC violating matter with respect to dimension for different $\phi_{0}(G=1$ ) .

\section{Acknowledgments:}

F.R is thankful to Jadavpur University and UGC , Government of India for providing financial support. 


\section{Appendix:}

The shape function for the case $d=2$ i.e. for the four dimensional spacetime is very similar to the case studied by Ellis [26]. But our work is interesting and is more justified as we have taken care of minimizing the total amount of exotic matter to be needed to construct the wormhole. In this case, one can get exact analytical forms of embedded function $\mathrm{z}$ and proper radial distance as

$Z= \pm \sqrt{4 \pi G \phi_{0}^{2}} \cosh ^{-1} \frac{r}{\sqrt{4 \pi G \phi_{0}^{2}}}$

$l(r)= \pm \sqrt{r^{2}-4 \pi G \phi_{0}^{2}}$

One can see embedding diagram of this wormhole in fig 9. The surface of revolution of this curve about the vertical $\mathrm{z}$ axis makes the diagram complete (fig10).



Figure 9: The embedding diagram of the wormhole $\left(\phi_{0}=.01\right.$ and $\left.G=1\right)$.

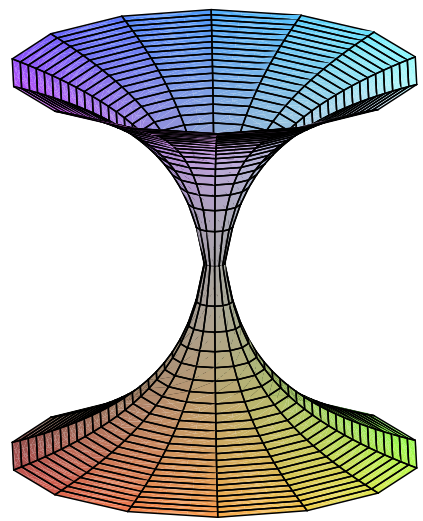

Figure 10: The full visualization of the surface generated by the rotation of the embedded curve about the vertical $\mathrm{z}$ axis .

Due to the simple expression for $l(r)$, one can rewrite the metric tensor in terms of this proper radial distance,

$d s^{2}=-d t^{2}+d l^{2}+r^{2}(l) d \Omega_{2}^{2}$,

where

$r^{2}(l)=l^{2}+4 \pi G \phi_{0}^{2}$

This is a well behaved coordinate system.

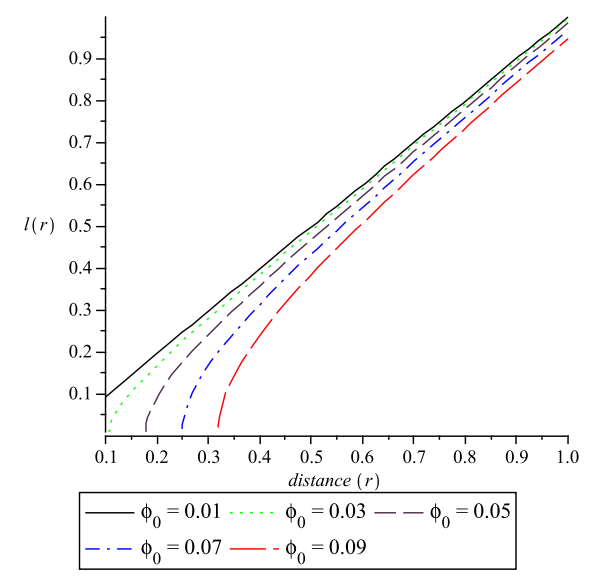

Figure 11: The diagram of the radial proper distance of the wormhole for different $\phi_{0}$ ( $G=1$ ). 


\section{References}

[1] S. Perlmutter et.al., astro-ph/971221

[2] A. G. Riess et.al, astro-ph/9805201

[3] M. Morris and K. Thorne , American J. Phys. 56, 39 (1988)

[4] M. C. Bento, O. Bertolami and A. A. Sen, Phys. Rev. D 66, 043507 (2002)

[5] A. Melchiorri, L. Mersini, C. J. Odman and M. Trodden, Phys. Rev. D 68 043509 (2003)

[6] S. M. Carroll, M. Hoffman and M. Trodden, Phys. Rev. D 68023509 (2003)

[7] H Stefancic, Phys. Rev. D 71124036 (2005)

[8] Francisco S.N. Lobo, Phys.Rev.D71:124022,2005

[9] Francisco S.N.Lobo, Phys.Rev.D 71 : 084011, 2005

[10] Sergey V.Sushkov, Phys.Rev.D 71: 043520, 2005.

[11] O.B. Zaslavskii , Phys.Rev.D 72: 061303, 2005.

[12] PeterK.F.Kuhfittig, Class.Quant.Grav. 23 : 5853-5860, 2006.

[13] F. Rahaman et al, Phys.Lett.B633:161163,2006

[14] F. Rahaman et al, Phys.Scr. 76:5659,2007 e-Print: arXiv:0705.1058 grqc]

[15] F. Rahaman et al, arxiv:gr-qc/0701032

[16] F. Rahaman et al, Gen.Rel.Grav.39:145-151,2007
[17] Francisco S.N.Lobo, Phys.Rev.D 73: 064028, 2006.

[18] F.Rahaman et al , Mod.Phys.Lett.A 23: 1199-1211, 2008.

[19] Subenoy Chakraborty, Tanwi Bandyopadhyay, arXiv:0707.1183 [gr-qc]

[20] Muneer Ahmad Rashid, Mubasher Jamil, Umar Farooq, arXiv:0809.3376 [gr-qc]

[21] V. M. Zhuravlev et al, General Relativity and Gravitation 36, 1719-1736 (2004)

[22] J Kujat et al, arxiv: astro-ph/0606735

[23] R J Scherrer et al, arxiv: 0808.1880 [astro-ph]

[24] M Visser, S Kar and N Dadhich, Phys.Rev.Lett.90:201102,2003

[25] F. Rahaman et al, Gen.Rel.Grav. 38 : 1687-1695, 2006.

[26] G. Ellis, J.Math.Phys. 14, 104 (1973) 